\title{
因HAD
}

ISSN-L: 2530-5115

(1) (1)(2)

DOI: http://doi.org/10.22585/hospdomic.v1i3.24

\section{Análisis bibliométrico de la producción científica española, indizada en MEDLINE, sobre servicios de atención a domicilio provisto por hospital}

Bibliometric analysis of Spanish scientific production indexed in MEDLINE, about hospital-based home care services

Andrea Domingo-Pueyo

Departamento de Salud Pública, Historia de la Ciencia y Ginecología de la Universidad Miguel Hernández de Elche, Alicante. España

Correspondencia/Correspondence

Andrea Domingo Pueyo

Avd Armada Española, 14 6E

03502 Benidorm (Alicante)

andreadp79.ad@gmail.com

+34687746877
Recibido/Received

26.06.2017

Aceptado/Accepted

05.07.2017

Conflicto de Intereses/Competing interest

La autora declara la inexistencia de cualquier tipo de conflicto de interés. 


\section{RESUMEN}

Objetivo: Analizar la producción científica española, indizada en MEDLINE, sobre servicios de atención a domicilio provisto por hospital.

Método: Estudio descriptivo transversal. Se calcularon y analizaron, mediante técnica bibliométrica, los principales indicadores sobre producción científica. La búsqueda se realizó en la base de datos bibliográfica MEDLINE, a través de PubMed, mediante la construcción de una ecuación final por unión booleana de los filtros previamente testados en la literatura científica. La fecha de realización de la búsqueda fue mayo de 2017.

Resultados: De la consulta realizada se obtuvieron un total de 105 referencias. El año más productivo fue el 2009 con 11 artículos. La tipología documental más frecuente fue el artículo original con 91 documentos $(96,7 \%)$ siendo el Índice de Productividad de 1,96. La evolución de las publicaciones fue ascendente, ajustándose a un modelo lineal, dando un coeficiente de determinación $R^{2}=0,96$ y $p<0,001$. La edad de los documentos analizados fue de 9,1 \pm 0,5 años, con mediana de 8 años. Se identificaron trabajos publicados de 73 instituciones como primer firmante (de un total de 99).

Conclusión: El artículo original es la principal tipología documental en la producción científica sobre Hospital a Domicilio con filiación española, con obsolescencia superior a la de otras áreas de las ciencias de la salud. La coautoría -media de autores firmantes- es baja, no existiendo grandes grupos de investigación interinstitucionales.

Palabras clave: Servicios de atención a domicilio provisto por hospital; Bibliometría; Acceso a la Información; Indicadores bibliométricos.

\section{ABSTRACT}

Aim: To analyze the Spanish scientific production, indexed in MEDLINE, about hospital-based home care services.

Method: Cross-sectional descriptive study. The main indicators on scientific production were calculated and analyzed, using bibliometric technique. The search was performed in the MEDLINE bibliographic database, through PubMed, by constructing a final equation by boolean union of the filters previously tested in the scientific literature. The date of the search was May 2017.

Results: A total of 105 references were obtained from the consultation. The most productive year was 2009 with 11 articles. The most frequent documentary typology was the original article with 91 documents (96.7\%), with the Productivity Index being 1.96. The evolution of the publications was upward, adjusting to a linear model, giving a coefficient of determination $R^{2}=0.96$ and $p<0.001$. The age of the documents analyzed was $9.1 \pm 0.5$ years, with a median of 8 years. We identified published papers from 73 institutions as the first signer (out of a total of 99).

Conclusion: The original article is the main documentary typology in the scientific production on home care services, hospital-based with Spanish affiliation, with obsolescence superior to that of other areas of the health sciences. The coauthor-media of signatory authors- is low, and there are no large inter-institutional research groups.

Keywords: Home care services, hospital-based; Bibliometrics; Access to information; Bibliometric indicators. 


\section{INTRODUCCIÓN}

El origen de la Hospitalización a Domicilio (HAD) no puede menos que contextualizarse con las necesidades sanitarias que primaban a lo largo del pasado siglo XX. La congestión de los hospitales y la escasez de camas de hospitalización marcaron nuestros orígenes y durante mucho tiempo se priorizó la descarga del hospital como el objetivo de referencia para la HAD. Sin embargo, en los últimos años estamos asistiendo a un progresivo proceso de "ambulatorización" de la gestión sanitaria, avances tecnológicos en materia diagnóstica y terapéutica que no hacen necesaria la ubicación del paciente en la estructura hospitalaria y una cronificación de los procesos médicos, ligada al envejecimiento de la población, que obliga a un cambio de paradigma en los modelos sanitarios que tienen en la hospitalización a domicilio como aliado necesario (1).

El crecimiento de esta modalidad asistencial debería extrapolarse al número de publicaciones y, por tanto, es esperable un crecimiento de los artículos científicos sobre HAD.

Un artículo científico es la suma coherente de sus componentes, un informe escrito que describe los resultados de una investigación. Desde hace unos años la mayor parte de las comunicaciones de resultados de las investigaciones se difunden mediante revistas, por lo que los estudios bibliométricos se presentan como herramientas fundamentales para caracterizarlas y evaluarlas (2). Así, el estudio de la producción científica mediante análisis bibliométrico permite examinar determinados aspectos relevantes sobre una temática científica concreta como por ejemplo: en qué revistas /plataformas se publica más, qué instituciones tienen mayor relevancia en el campo referido, o qué publicaciones son más visibles frente al ruido generado por la competitividad del mercado laboral, qué grupos de investigación son los más destacados, además de medir la obsolescencia y dispersión de las publicaciones (3).

El crecimiento de la producción científica en las últimas décadas y su indización en bases de datos bibliográficas automatizadas han potenciado el uso de la bibliometría y la generación de indicadores para medir los resultados de la actividad científica y tecnológica (4). También, la producción científica de un área temática determinada sigue siendo un buen indicador de la progresión de la investigación y de la generación de conocimientos. Su análisis bibliométrico permite un examen retrospectivo sobre el modo que ha sido investigada y dada a conocer, pero también puede evaluar el potencial de investigación de las instituciones involucradas (5).

Cuando se considera aisladamente la bibliometría se podría argumentar que es altamente reduccionista, que no es representativa del rendimiento o la capacidad de un investigado; sin embargo, tiene una gran utilidad porque en términos pragmáticos representa una fuente de datos precisos y puede evaluar el potencial de investigación de los grupos e instituciones involucradas (4). El cálculo de indicadores bibliométricos no puede limitarse a la aportación de datos estadísticos, sin más y por separado, sino que deben estar relacionados para que puedan aportar una explicación sólida sobre la actividad científica que se esté considerando. Por tanto, para relacionar los indicadores con el área de conocimiento específica es necesario encajar el cálculo métrico con el análisis temático (6).

Por todo lo anteriormente expuesto, queda patente la necesidad del objeto a estudio: llevar a cabo un análisis bibliométrico de la producción científica española, indizada en MEDLINE, sobre hospitalización a domicilio. 


\section{MÉTODOS}

\section{Diseño}

Estudio descriptivo transversal de la producción científica española sobre hospitalización a domicilio.

\section{Fuente de obtención de datos}

Se obtuvieron de la consulta directa y acceso, vía Internet, a esta literatura científica recogida en la base de datos MEDLINE, vía PubMed.

\section{Unidad de análisis}

Se trabajó con los artículos publicados y recuperados desde la base de datos bibliográficos indicada.

\section{Búsqueda bibliográfica}

Ecuación 1: Para construir la ecuación de búsqueda sobre hospitalización a domicilio fue la publicada por Sanz-Valero et al. (4).

Ecuación 2: La ecuación empleada en el filtro geográfico español es la desarrolla por SanzValero et al. (7).

Ecuación final: Ecuación 1 AND Ecuación 2

La fecha de realización de la búsqueda fue mayo de 2017.

\section{Indicadores a estudio}

- Producción científica, calculada según número de artículos indizados.

- Tipología documental y número de artículos citables (suma de artículos de investigación original y de revisión).

- Índice de productividad (logaritmo del número de trabajos originales publicados).

- Edad: 2017 menos año de publicación del artículo.

- Semiperiodo de Burton-Kebler (Mediana de la distribución del conjunto de las referencias ordenadas por su antigüedad).

- Índice de Price (porcentaje de referencias con edad menor de 5 años).

- Idioma de publicación del artículo

- Índice de colaboración institucional: Número de instituciones que figuran como firmantes del documento.

- Filiación institucional del primer firmante e Índice de Lotka. Las instituciones se agruparon en tres niveles de rendimiento: pequeños productores (Índice de transitoriedad = un único trabajo), medianos productores (entre 2 y 9 trabajos) y grandes productores (10 o más trabajos).

- Número de autores por artículo e índice de colaboración (cociente entre el número de firmas y el número de trabajos).

- Revista donde se publica el artículo.

- Dispersión: núcleo principal de Bradford (conjunto de revistas de mayor pertinencia para un área del conocimiento).

- Enlace: Existencia de enlace al documento desde PubMed. 
- Acceso: Posibilidad de acceder al texto completo del artículo gratuito a través de del enlace existente en PubMed.

\section{Análisis de los datos}

Las variables cualitativas se describieron por su frecuencia absoluta y relativa (porcentaje), las cuantitativas mediante su Media y Desviación Estándar, representando las más relevantes mediante la utilización de tablas y gráficos. Se utilizó la Mediana, como medida de tendencia central y se calculó el rango para cada variable pertinente.

El crecimiento de la producción científica se examinó mediante el análisis de regresión. El nivel de significación utilizado en todos los contrastes de hipótesis fue $a \leq 0,05$.

Para el almacenamiento y análisis estadístico se utilizó el programa Statistical Package for the Social Sciences (IBM-SPSS), versión 22 para Windows. El control de la calidad de la información se efectuó mediante la doble entrada de los datos, corrigiendo las inconsistencias mediante la consulta con los datos originales.

\section{RESULTADOS}

\section{Producción científica}

De la consulta realizada en la base de datos MEDLINE se obtuvieron un total de 105 referencias.

El año más productivo fue el 2009 con 11 artículos, un 10,5\% del total de la producción sobre hospitalización a domicilio. Los dos primeros artículos indizados en la base de datos de referencia fueron publicados en las revistas Medicina Clínica (8) y Atención Primaria (9) en 1997.

La evolución de las publicaciones fue ascendente a lo largo de los años, ajustándose a un modelo lineal, dando un coeficiente de determinación $R^{2}=0,96$ y $p<0,001$, ver figura 1 .

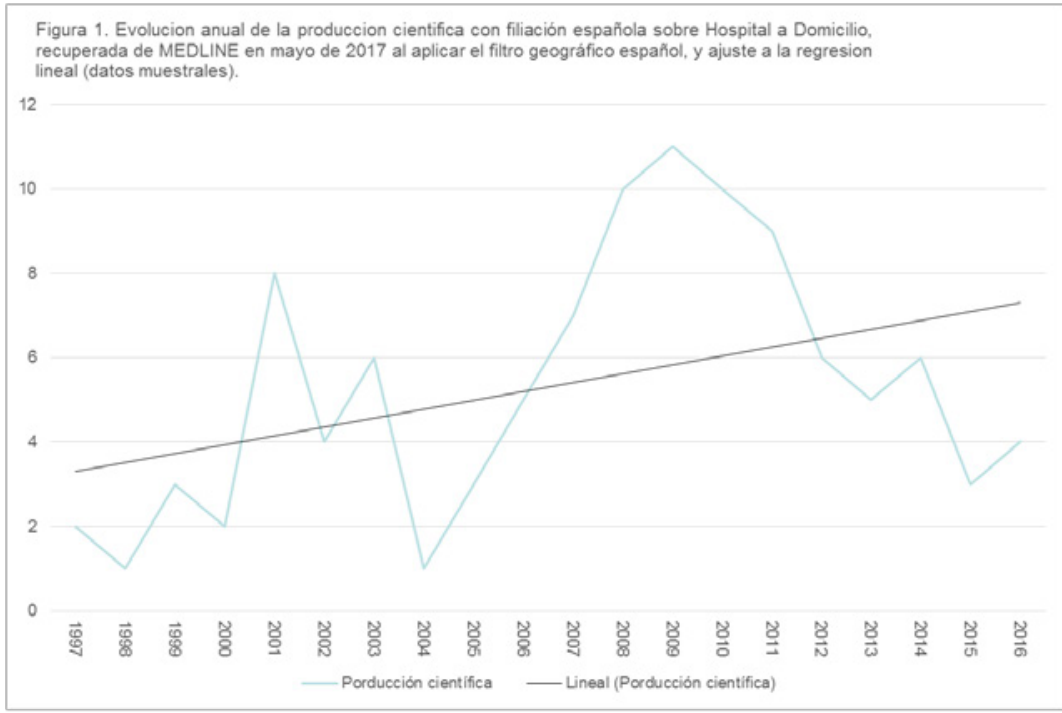




\section{Tipología documental}

La tipología documental más frecuente fue el artículo original con 91 documentos (86,7\%), el número de revisiones fue de $11(10.5 \%)$ y el de cartas, de $3(2.9 \%)$, ver tabla 1.

El porcentaje de la suma de artículos de investigación original y de revisión, computables para el cálculo del impacto -los denominados artículos citables-, fue de 97,1\%.

El índice de productividad general de artículos de investigación original fue de 1,96.

\begin{tabular}{|c|c|c|}
\hline Tipo de artículo & Frecuencia & Porcentaje (\%) \\
\hline Original & 91 & 86,7 \\
\hline Revisión & 11 & 10,5 \\
\hline Carta & 3 & 2,9 \\
\hline Total & 105 & 100,0 \\
\hline
\end{tabular}

\section{Edad de los artículos y obsolescencia/actualidad de la producción científica}

La edad media de las referencias analizadas, fue de 9,1 \pm 0,5 años, con mediana de 8 (Semiperiodo de Burton-Kebler) y máximo 20 años. El índice de Price calculado fue del 17,1\%.

\section{Idioma, procedencia geográfica y filiación institucional}

El idioma predominante de las referencias recuperadas fue el español, con 60 casos $(57,1 \%)$. El inglés fue un idioma en el que ampliamente también se publicó (44; 41,9\%) y en francés únicamente se publicó un artículo (1,0\%) (10). Ver Tabla 2.

\begin{tabular}{|c|c|c|}
\hline Idioma de publicación & Frecuencia & Porcentaje (\%) \\
\hline Español & 60 & 57,1 \\
\hline Inglés & 44 & 41,9 \\
\hline Francés & 1 & 1,0 \\
\hline Total & 105 & 100,0 \\
\hline
\end{tabular}


En el conjunto de la búsqueda se identificaron documentos de 3 países de filiación de institución diferentes, siendo España el de mayor producción con 103 referencias (98,1\%). Ver Tabla 3.

\begin{tabular}{|l|r|r|}
\hline \multicolumn{2}{|c|}{ Tabla 3. País de filiación de los artículos sobre Hospital a Domicilio indizada en MEDLINE } \\
\hline País de filiación & Frecuencia & Porcentaje (\%) \\
\hline España & 103 & 98,1 \\
\hline Alemania & 1 & 1,0 \\
\hline Francia & 1 & 1,0 \\
\hline Total & 105 & 100,0 \\
\hline
\end{tabular}

Se identificaron trabajos publicados de 73 instituciones como primer firmante (de un total de 99), con una Mediana de 1 centro por artículo. La media del número de instituciones por artículo -índice de colaboración institucional- fue de 1,4 \pm 0,1. Ver Tabla 4.

\begin{tabular}{|l|r|r|}
\hline \multicolumn{2}{|l|}{ Tabla 4. Institución de filiación del $1^{\circ}$ autor que han publicado más de 2 artículos sobre Hospital a } \\
Domicilio indizada en MEDLINE & Frecuencia & Porcentaje (\%) \\
\hline Institución de filiación (1er autor) & 7 & 6,7 \\
\hline Vall d'Hebrón, Hospital & 5 & 5,7 \\
\hline San Carlos, Hospital Clínico & 5 & 4,8 \\
\hline Barcelona, Hospital Clínic & 4 & 4,8 \\
\hline Txagorritxu, Hospital & 3 & 3,8 \\
\hline Gregorio Marañón, Hospital & 3 & 2,9 \\
\hline Barcelona SCIAS, Hospital & 3 & \\
\hline Badalona, Hospital Municipal & & \\
\hline
\end{tabular}

Un total de 15 estudios (14,3\%) fueron realizados en colaboración entre 2 o más centros.

Las instituciones se clasificaron en tres niveles de rendimiento extrapolando a las mismas el Índice de Lotka: índice de transitoriedad: 84 ( $84,8 \%)$ eran pequeños productores ( 1 trabajo publicado), $15(15,2 \%)$ eran medianos productores (entre 2 y 10 trabajos) y no se observó ninguna institución con más de 10 trabajos. Ver Tabla 5. 


\begin{tabular}{|c|c|c|}
\hline Instituciones de filiación ( $\geq 5$ artículos) & Frecuencia & Porcentaje (\%) \\
\hline Vall d'Hebrón, Hospital & 8 & 5,6 \\
\hline San Carlos, Hospital Clínico & 6 & 4,2 \\
\hline Barcelona, Hospital Clínic & 5 & 3,5 \\
\hline Txagorritxu, Hospital & 5 & 3,5 \\
\hline Gregorio Marañón, Hospital & 5 & 3,5 \\
\hline
\end{tabular}

\section{Autoría}

Se contabilizaron un total de 564 autores, con un máximo de 21 autores observado en un solo artículo. La media, equivalente al Índice de Cooperación, fue de 5,4 \pm 0,3 autores. La mediana fue de 5 autores por artículo.

\section{Revistas, impacto y dispersión de la literatura científica}

A partir de la muestra seleccionada se recuperaron un total de 5 revistas, de las que componían el núcleo principal de Bradford, ver figura 1. Como puede observarse en la Tabla 6, las revistas que publicaron 5 o más artículos sobre el tema a estudio fueron 4.

\begin{tabular}{|c|c|c|}
\hline Revistas ( $\geq 5$ artículos filiación española) & Frecuencia & Porcentaje (\%) \\
\hline Med Clin (Barc) & 13 & 12,4 \\
\hline Arch Bronconeumol & 6 & 5,7 \\
\hline Eur J Intern Med & 6 & 5,7 \\
\hline Rev Clin Esp & 5 & 4,8 \\
\hline
\end{tabular}

El estudio de la dispersión de la literatura científica recuperada, determina la concentración de una frecuencia similar de documentos en un número diferente de revistas: núcleo principal, con 5 revistas (8,5\%) que incluyen 34 artículos (32,4\%). El núcleo 2 con 18 revistas (30,5\%) que contienen 
36 artículos (33,3\%) y el núcleo 3 con 36 revistas (61,0\%) que engloban 36 artículos (34,3\%); ver figura 2: gráfico de dispersión según Bradford.

Figura 2: Dispersión de las revistas y los artículos con filiación española sobre Hospital a Domicilio en la base de datos bibliográfica MEDLINE (mayo 2017)

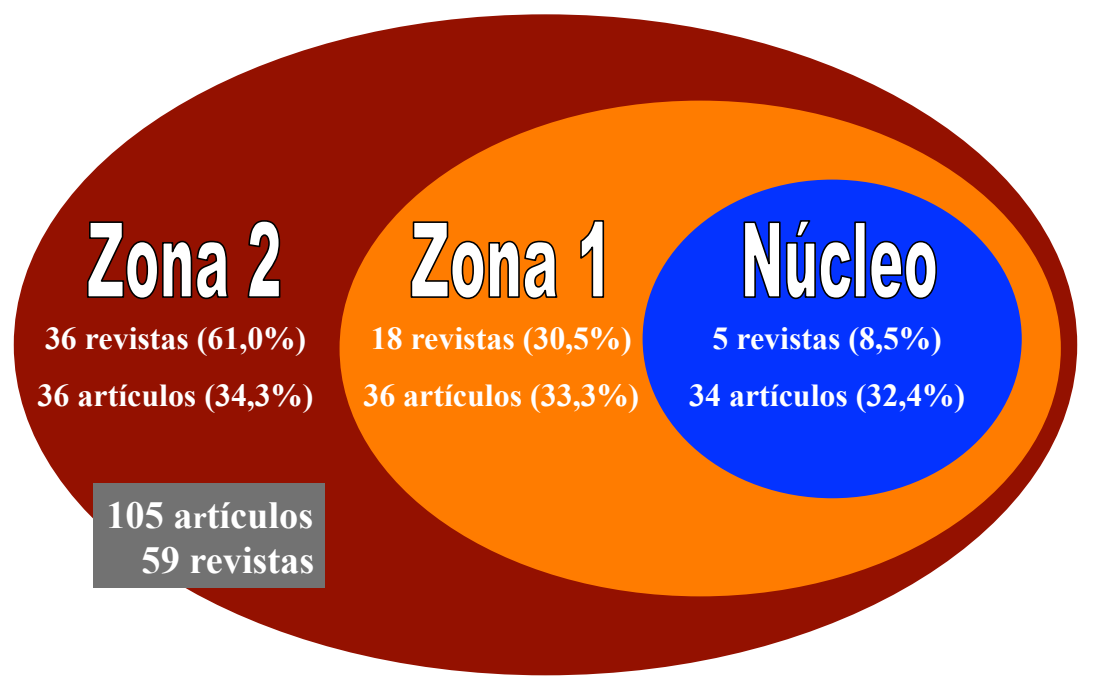

\section{Acceso al documento primario}

En relación al enlace al texto completo desde MEDLINE se comprobó que se pudo acceder a él en 85 ocasiones $(81,0 \%)$ siendo en 44 ocasiones $(41,9 \%)$ de forma gratuita. Los datos perdidos corresponden con el número de enlace rotos, que asciende a 7 (6,67\%).

\section{DISCUSIÓN}

Este trabajo presenta los principales indicadores bibliométricos de la producción científica española sobre Hospital a Domicilio indizada en la base de datos bibliográfica MEDLINE. Teniendo en cuenta los resultados obtenidos los documentos recuperados están en consonancia con la temática seleccionada.

En la evolución de la producción científica se observa un progresivo incremento que no alcanza el crecimiento exponencial, en contraposición con las teorías cienciométricas, que anuncian modelos exponenciales aunque para tal cumplimiento se necesitan periodos temporales superiores a los 30 años.

En relación a la tipología documental observada, no es de extrañar el predominio de los artículos originales al tratarse de un área de estudio con aplicación eminentemente clínica. En consecuencia, el Índice de Productividad obtenido es similar al observado es otros estudios bibliométricos sobre las ciencias de la salud (11). Asimismo, y por la misma causa, el número de artículos citables se considera muy adecuado (12). 
El análisis de la actualidad/obsolescencia, medido tanto por el Índice de Burton-Kebler como por el Índice de Price, presenta resultados que penalizan los indicadores del tema a estudio al ser superiores a los datos en el área de las ciencias de la salud (13).

El predominio del idioma español se debe al enfoque de este trabajo, la filiación española, por lo que no debe compararse con estudios métricos del ámbito general donde el inglés hubiera sido el idioma predominante (14).

El indicador sobre colaboración institucional no muestra la existencia de grandes grupos de investigación y el Índice de Colaboración, entre autores, presenta resultados inferiores a los publicados en el mismo ámbito de conocimiento de la salud $(15,16)$. Estos resultados pueden disminuir la visibilidad y el impacto de la materia general estudiada y no contribuir a fomentar el desarrollo del conocimiento, sobre Hospital a Domicilio con filiación española, como elemento central.

En cuanto al acceso al texto completo, de forma libre, permanente y gratuita, se comprueba el avance de la iniciativa Open Access acorde con los principales movimientos de apertura de la transmisión del conocimiento, aunque en este sentido queda mucho por trabajar (17).

Como posibles limitaciones, señalar la existencia de una gran producción científica española que no se encuentra indizada en MEDLINE. Igualmente, se conoce que en MEDLINE existen limitaciones de campo, en las que hasta el año 2013, sólo se recoge la dirección del primer autor, hecho que conduce una restricción en la obtención de una mayor información (18).

Si bien se ha utilizado el autor designado para la correspondencia para estudiar la filiación institucional y esto puede ir en detrimento del resto de los autores del artículo, y ser posible sesgo. Existen trabajos que han analizado la no existencia de diferencias significativas entre tomar sólo este autor o la totalidad de ellos (4).

Por todo lo anteriormente expuesto se puede concluir que el artículo original es la principal tipología documental en la producción científica sobre Hospital a Domicilio con filiación española, con obsolescencia superior a la de otras áreas de las ciencias de la salud. La coautoría -media de autores firmantes- es baja, no existiendo grandes grupos de investigación interinstitucionales.

\section{BIBLIOGRAFÍA}

1. Massa Domínguez B. La hospitalización a domicilio en el siglo XXI. Hosp Domic. 2017;1(1):7. DOI: doi.org/10.22585/hospdomic.v111.8

2. Tomás- Casterá V, Sanz-Valero J, Wanden-Berghe. Estudio bibliométrico de la producción científica de la revista de Nutriçao a través de la Red SciELO (2001 a 2007). Rev Nut. 2010;23(5):7919. DOI: 10.3305/nh.2013.28.3.6463; PMID: 23848128.

3. Palomo-Llinares B, Palomo-Llinares R, Sanz-Valero J. Análisis bibliométrico de los sistemas de medicación hospitalarios. Ars Pharm. 2017;58(1):29-37. DOI:10.4321/S2340-98942017000100004

4. Sanz-Valero J, Wanden-Berghe C. Análisis bibliométrico de la producción científica, indizada en MEDLINE, sobre los servicios de salud proporcionados por las unidades de hospitalización a domicilio. Hosp Domic. 2017;1(1):21. DOI: doi.org/10.22585/hospdomic.v111.3

5. Sanz-Valero J. Estudio bibliométrico de producción y consumo de la revista Farmacia Hospitalaria (2004-2012). Farm Hosp. 2014; 38(1):1-8. DOI: 10.7399/ FH.2014.38.1.1153; PMID: 24483853.

6. Quesada-Risueño P, Sanz-Valero J, Wanden-Berghe C. Análisis bibliométrico de la producción científica existente en la base de datos bibliográfica MEDLINE sobre la fibra dietética. Rev Esp Nutr Humana Dietética. 2017;21(1):29-38. DOI: 10.14306/renhyd.21.1.275 
7. Sanz-Valero J, Veiga de Cabo J, Rojo-Alonso C, D'Agostino MJ, Wanden-Berghe, Espulgues Pellicer JX, et al. Los filtros metodológicos: aplicación a la búsqueda bibliográfica en la medicina del trabajo española. Med Segur Trab. 2008;LIV(211):75-83.

8. Oterino de la Fuente D, Ridao M, Peiró S, Marchan C. [Hospital at home and conventional hospitalization. An economic evaluation]. Med Clin (Barc). 1997;109(6):207-11. PMID: 9289549.

9. Lamelo Alfonsín F, Silva César M, Aldámiz-Echevarría Iraurgui B, García Doval A. [Evaluation of the experience in domiciliary hospitalization of trauma patients]. Aten Primaria. 1997;20(4):1957. PMID: 9410143

10. Romain D, Bernady A, Etchamendy E, Barokas T, Pignede P. [Costs of hospitalisation for exacerbations of COPD in patients receiving domiciliary rehabilitation]. Rev Mal Respir. 2011;28(7):86472. DOI: 10.1016/j.rmr.2011.06.001; PMID: 21943531.

11. Sanz-Valero J, Gil A, Wanden-Berghe, Martínez de Victoria E, Grupo de comunicación y documentaicón científica en nutrición (CDC-Nut SENPE). Análisis bibliométrico y temático de la producción científica sobre ácidos grasos omega-3 indizada en las bases de datos internacionales sobre ciencias de la salud. Nutr Hosp. 2012;27(Supl.2):41-8. DOI: 10.3305/nh.2012.27. sup2.6272; PMID: 23568396

12. Aleixandre Benavent R, Valderrama Zurián JC, Castellano Gómez M, Simó Meléndez R, Navarro Molina C. Factor de impacto de las revistas médicas españolas. Med Clin (Barc). 2004;123(18):697-701. PMID: 15563817.

13. Wanden-Berghe C, Martín-Rodero H. 25 años de investigación en nutrición y alimentación en el espacio iberoamericano del conocimiento. Nutr Hosp. 2012;27(Supl 2):26-33. DOI: 10.3305/ nh.2012.27.sup2.6270; PMID: 23568394

14. Franco-López A, Sanz-Valero J, Culebras JM. Publicar en castellano, o en cualquier otro idioma que no sea inglés, negativo para el factor de. J Negat No Posit Results. 2016;(2):65-70. DOI: 10.19230/jonnpr.2016.1.2.1005

15. Tomás-Casterá V, Sanz-Valero J, Juan-Quilis V. Estudio bibliométrico de la producción científica y de consumo de las revistas sobre nutrición indizadas en la red SciELO. Nutr Hosp. 2013;28(3):969-70. DOI: 10.3305/nh.2013.28.3.6463; PMID: 23848128

16. Camargo Jr KR de, Coeli CM. Multiple authorship: growth or inflationary bubble? Rev Saúde Pública. 2012;46(5):894-900. PMID: 23128267

17. Quesada-Risueño P, Sanz-Valero J, Wanden-Berghe C. Análisis bibliométrico de la producción científica existente en la base de datos bibliográfica MEDLINE sobre la fibra dietética. Rev Esp Nutr Humana Dietética. 2017;21(1):29. DOI: 10.14306/renhyd.21.1.275

18. Sanz-Valero J, Veiga de Cabo J, Rojo-Alonso C, D’Agostino MJ, Wanden-Berghe, Espulgues Pellicer JX, et al. Los filtros metodológicos: aplicación a la búsqueda bibliográfica en la medicina del trabajo española. Med Segur Trab. 2008;LIV(211):75-83. 